\title{
Comentarios del artículo: “Niveles de sedentarismo de una institución educativa en Popayán, Colombia"
}

Sr. Editor, recientemente leímos con interés el artículo publicado en la Revista Universidad y Salud en su Volumen 21, número 3, denominado "Niveles de sedentarismo de una institución educativa en Popayán, Colombia, cuyo objetivo fue "determinar los niveles de sedentarismo de los estudiantes de una Institución Educativa de PopayánColombia"(1). Felicitamos a los autores por el gran valor y alta pertinencia del artículo en general, cuyos resultados contribuyen a elevar el nivel de conocimiento respecto a los niveles de sedentarismo relacionado a variables sociodemográficas, antropométricas y estilos de vida en niños y adolescentes, sin embargo, con el objetivo de aportar mayor e importante información nos gustaría exponer algunas reflexiones y complementar la información entregada a partir de los resultados, la discusión que se despliega del trabajo y la conclusión de este. Para realizar nuestros comentarios, nos permitimos en la Tabla 1, describir la clasificación para el Índice de Masa corporal (IMC), Circunferencia de Cintura (CC) e Índice Cintura/Cadera (ICC) según estudios de la Asociación de la Fuerza Prensil con Manifestaciones Tempranas de Riesgo Cardiovascular en Niños y Adolescentes Colombianos (FUPRECOL) sobre valores referenciales para escolares de Bogotá-Colombia ${ }^{(2,3)}$.

Tabla 1. Distribución media del Índice de Masa Corporal, Circunferencia de Cintura e Índice Cintura/ Cadera según sexo y edad

\begin{tabular}{clccc}
\hline Niños y adolescentes & $\mathbf{n}$ & IMC $\mathbf{( k g / \mathbf { m } ^ { 2 } )}$ & CC $(\mathbf{c m})$ & ICC \\
\hline 9 a 17,9 años & 2.542 & $19,3 \pm 3,4^{*}$ & $66,2 \pm 8,2^{*}$ & $0,82 \pm 0,10^{*}$ \\
\hline Niñas y adolescentes & $\mathbf{n}$ & IMC $\mathbf{( k g / \mathbf { m } ^ { 2 } )}$ & $\mathbf{C C}(\mathbf{c m})$ & ICC \\
\hline 9 a 17,9 años & 3.379 & $19,9 \pm 3,6$ & $65,2 \pm 8,2$ & $0,78 \pm 0,06$ \\
\hline
\end{tabular}

${ }^{*} p<0,01$. Diferencias por grupo de edad y sexo.

Chalapud-Narváez et al.,(1) en su análisis estadístico no encontró relación entre los niveles de sedentarismo y las variables sociodemográficas, antropométricas y de estilos de vida, concluyendo en base al análisis descriptivo que la población escolar presenta un factor de riesgo para la salud asociado a comportamientos sedentarios. Respecto a estas conclusiones, nos gustaría recordar que el análisis antropométrico en base al IMC posee una baja sensibilidad para reflejar cambios del tejido adiposo en adolescentes, por lo que, con el objetivo de evitar errores de estimación en la determinación del estado nutricional es necesario utilizar medidas de composición corporal complementarias considerando variables como el sexo, edad, madurez sexual y país de residencia de la población(4).

En este contexto, creemos acertado el uso del puntaje de desvío estándar o Z-score(1), sin embargo, consideramos que el análisis y discusión de los resultados pudo ser complementado con tablas específicas para la población de estudio como las proporcionadas por los estudios FUPRECOL(2,3). Siguiendo esta línea, la importancia de estos estudios radica en que determinaron el estado nutricional y riesgo cardiovascular en edades tempranas destacando; el mayor IMC en niñas en comparación a niños colombianos, mientras que los niños reportaron mayores resultados en la CC e ICC. Por otro lado, al comparar estos resultados con otros países se puede apreciar que la CC fue menor a la reportada en Perú e Inglaterra con excepción de India, Venezuela, Estados Unidos y España, del mismo el ICC presenta valores menores en comparación a Europa, Suramérica, Asia y África, no así para Grecia y Venezuela ${ }^{2,3}$. 
Finalmente, nos parece necesario que Chalapud-Narváez et al.,(1) aclaren si usaron o no un instrumento estandarizado para la medición y registro del peso y talla, además del por qué no complementaron la información entregada por el Z-score con medidas de composición corporal de mayor especificidad para la población estudiada.

Catalina González-Wong' orcid.org/0000-0003-0360-8567

Héctor Fuentes-Barría ${ }^{2 *}$ orcid.org/0000-0003-0774-0848

Raúl Aguilera-Eguía ${ }^{3}$ orcid.org/0000-0002-4123-4255

Sebastián Urbano-Cerda ${ }^{2}$ orcid.org/0000-0003-0508-6985

Valentina Vera-Aguirre 4 orcid.org/0000-0003-3050-5192

1. Enfermería, Facultad de Medicina, Universidad del Desarrollo. Santiago, Chile.

2. Escuela de Ciencias del Deporte y Actividad Física, Facultad de Salud, Universidad Santo Tomás, Chile.

3. Departamento de Salud Pública, Facultad de Medicina, Carrera de kinesiología. Universidad Católica de la Santísima Concepción. Concepción, Chile

4. Escuela de Nutrición y Dietética, Facultad de Ciencias para el Cuidado de la Salud, Universidad San Sebastián, Chile

Conflictos de interés: No declaran.

Fecha de recepción: Enero 03 - 2020

Fecha de revisión: Septiembre 20 - 2020

\author{
*Autor de correspondencia \\ Héctor Fuentes-Barría \\ e-mail: hectorfuentesbarria@gmail.com
}

Fecha de aceptación: Abril 29 - 2021

\section{Referencias}

1. Chalapud-Narváez L, Molano-Tobar N, Ordoñez-Fernández M. Niveles de sedentarismo de una institución educativa en Popayán, Colombia. Univ. Salud. 2019;21(3):198-204. doi: 10.22267/rus.192103.156

2. Caicedo-Álvarez J, Correa-Bautista JE, González-Jiménez E, Schmidt-RioValle J, Ramírez-Vélez R. Percentiles de circunferencia de cintura en escolares de Bogotá (Colombia): Estudio FUPRECOL. Endocrinología y Nutrición. 2016;63(6):265-73. doi: 10.1016/j.endonu.2016.01.008

3. Rodríguez-Bautista YP, Correa-Bautista EJ, González-Jiménez E, Schmidt-RíoValle J, Ramírez-Vélez R. Valores del índice cintura/cadera en la población escolar de Bogotá, Colombia: estudio FUPRECOL. Nutr. Hosp. 2015;32(5):2054-61. doi: 10.3305/nh.2015.32.5.9633

4. Curilem-Gatica C, Almagià-Flores A, Rodríguez-Rodríguez F, Yuing-Farias T, Berral de la Rosa F, Martínez-Salazar C, et al. Evaluación de la composición corporal en niños y adolescentes: directrices y recomendaciones. Nutr. Hosp. 2016;33(3):734-8. doi: $10.20960 /$ nh.285 